\title{
Protective Effects of Kaempferol against Myocardial Ischemia/Reperfusion Injury in Isolated Rat Heart via Antioxidant Activity and Inhibition of Glycogen Synthase Kinase-3 $\beta$
}

\author{
Mingjie Zhou, ${ }^{1,2}$ Huanhuan Ren, ${ }^{3}$ Jichun Han, ${ }^{3}$ Wenjuan Wang, \\ Qiusheng Zheng, ${ }^{3,4}$ and Dong Wang ${ }^{1}$ \\ ${ }^{1}$ Shandong Provincial Qianfoshan Hospital, Jinan 250014, China \\ ${ }^{2}$ Weifang Medical University, Weifang 261031, China \\ ${ }^{3}$ Binzhou Medical University, Yantai 264003, China \\ ${ }^{4}$ Pharmacy School, Shihezi University, Shihezi 832002, China
}

Correspondence should be addressed to Qiusheng Zheng; zqsyt@sohu.com and Dong Wang; wangdong9859@sina.com

Received 23 September 2014; Revised 9 November 2014; Accepted 11 November 2014

Academic Editor: Yanfang Chen

Copyright (C) 2015 Mingjie Zhou et al. This is an open access article distributed under the Creative Commons Attribution License, which permits unrestricted use, distribution, and reproduction in any medium, provided the original work is properly cited.

Objective. This study aimed to evaluate the protective effect of kaempferol against myocardial ischemia/reperfusion (I/R) injury in rats. Method. Left ventricular developed pressure (LVDP) and its maximum up/down rate $\left( \pm d p / d t_{\max }\right)$ were recorded as myocardial function. Infarct size was detected with 2,3,5-triphenyltetrazolium chloride staining. Cardiomyocyte apoptosis was determined using terminal deoxynucleotidyl nick-end labeling (TUNEL). The levels of creatine kinase (CK), lactate dehydrogenase (LDH), malondialdehyde (MDA), superoxide dismutase (SOD), glutathione/glutathione disulfide (GSH/GSSG) ratio, and tumor necrosis factor-alpha (TNF- $\alpha$ ) were determined using enzyme linked immunosorbent assay (ELISA). Moreover, total glycogen synthase kinase-3 $\beta$ (GSK-3 $\beta$ ), phospho-GSK-3 $\beta$ (P-GSK-3 $\beta$ ), precaspase-3, cleaved caspase-3, and cytoplasm cytochrome C were assayed using Western blot analysis. Results. Pretreatment with kaempferol significantly improved the recovery of LVDP and $\pm d p / d t_{\max }$, as well as increased the levels of SOD and P-GSK-3 $\beta$ and GSH/GSSG ratio. However, the pretreatment reduced myocardial infarct size and TUNEL-positive cell rate, as well as decreased the levels of cleaved caspase-3, cytoplasm cytochrome C, CK, LDH, MDA, and TNF- $\alpha$. Conclusion. These results suggested that kaempferol provides cardioprotection via antioxidant activity and inhibition of GSK-3 $\beta$ activity in rats with $\mathrm{I} / \mathrm{R}$.

\section{Introduction}

Nowadays, cardiovascular diseases are responsible for the majority of elderly mortality [1]; the most important presentation of cardiovascular disease is ischemia. A long period of ischemia leads to myocardial injury. Theoretically, restoring blood supply to the ischemic myocardium can reduce myocardial injury. However, reperfusion can aggravate myocardial damage through ischemia-reperfusion (I/R) injury [2]. Excessive reactive oxygen species (ROS), calcium overload, inflammatory reaction, and other factors can lead to cellular necrosis, apoptosis, and organ dysfunction in severe cases [3]. Prevention of I/R is important to alleviate ischemic heart disease [4].

Network pharmacology is a method for treating polygenic diseases based on the target and drug perspectives. Mapping the polypharmacology network onto the human disease-gene network can reveal important drug targets that could demonstrate the mechanism of botanical drugs in treating different diseases. Our previous studies identified the potential targets of kaempferol in cardiovascular diseases [5]. Thirteen potential targets were identified and annotated to have significant relationships with the pharmacologic effects of kaempferol. Among these targets, the main protein 
involved in I/R injury is glycogen synthase kinase-3 beta $(\mathrm{GSK}-3 \beta)$. GSK-3 $\beta$ is a serine/threonine kinase that participates in various cell activities through phosphorylation of the substrate protein [6]. GSK-3 $\beta$ is important in glycogen metabolism, as well as in cell proliferation, growth, and death $[7,8]$. GSK-3 $\beta$ has received increasing attention because of its involvement in some common and serious diseases, such as neurological disease, cancer, and I/R injury. In the cardiovascular system, GSK-3 $\beta$ has major roles in glucose metabolism, cardiomyocyte hypertrophy [9], and cell death [10]. Many studies have shown that GSK-3 $\beta$ inhibition during I/R is an important mechanism of myocardial adaptation; cardioprotective agents use the inhibition of GSK-3 $\beta$ (phosphorylation) as the common downstream target [11], and protection is related to the mitochondrial permeability transition pore (mPTP) [12].

Epidemiological studies have demonstrated that some flavonoids may affect treatment for several diseases [13]. A research on the plants used in traditional medicines revealed that flavonoids are their common bioactive constituents [14]. The flavonoid kaempferol, a yellow compound with low molecular weight (MW: $286.2 \mathrm{~g} / \mathrm{mol}$ ), is commonly found in plant-derived foods and in plants used in traditional medicines. Numerous preclinical studies have shown that kaempferol has a wide range of pharmacological activities, including antioxidant [15], anti-inflammatory [16], and anticancer activities [17].

Therefore, we aimed to evaluate the cardioprotective effects of kaempferol and the mechanisms underlying such effects in the present study.

\section{Methods}

2.1. Animals and Reagents. All procedures were performed in accordance with the National Institutes of Health Guideline on the Use of Laboratory Animals and were approved by the Shihezi University Committee on Animal Care. Adult male Sprague-Dawley rats (250-280 g) were obtained from the Xinjiang Medicine University Medical Laboratory Animal Center (SDXK 2011-004) and housed in a room with temperature of $22-25^{\circ} \mathrm{C}$, relative humidity of $50-60 \%$, and a 12-h light/12-h dark cycle.

Kaempferol (purity $\geq 98 \%$ ) was purchased from Shanghai Lichen Biotechnology Co., Ltd. (Shanghai, China). Antibodies against total GSK- $3 \beta$, as well as P-GSK-3 $\beta$ (Ser9), caspase3 , and cytoplasm cytochrome $\mathrm{C}$, were obtained from Cell Signaling Technology (1:10000, Beverly, MA, USA). Terminal deoxynucleotidyl nick-end labeling (TUNEL) assay was conducted using in situ cell death detection kit (POD, Roche, Germany). All other reagents were of standard biochemical quality and were obtained from commercial suppliers.

2.2. Establishment of Animal Model of Myocardial I/R Injury. The rats were randomly divided into four groups as follows: control group, I/R group, kaempferol group, and TDZD8 (4-benzyl-2-methyl-1,2,4-thiadiazolidine-3,5-dione) group. Hearts from control group were perfused for $120 \mathrm{~min}$ stabilization. Hearts from I/R group were stabilized for $20 \mathrm{~min}$ and then subjected to $15 \mathrm{~min}$ of global ischemia and $85 \mathrm{~min}$ of reperfusion. Hearts in kaempferol group were treated with $\mathrm{K}-\mathrm{H}$ buffer containing kaempferol $(15 \mathrm{mmol} / \mathrm{L})$ for $10 \mathrm{~min}$ after being stabilized and then subjected to global ischemia for $15 \mathrm{~min}$ and reperfusion for $85 \mathrm{~min}$. Hearts in TDZD8 group were treated with $\mathrm{K}-\mathrm{H}$ buffer containing TDZD$8(0.01 \mathrm{mmol} / \mathrm{L})$ for $10 \mathrm{~min}$ after being stabilized and then subjected to global ischemia for $15 \mathrm{~min}$ and reperfusion for $85 \mathrm{~min}$.

2.3. Heart Isolation and Perfusion. Rats were anesthetized with an intraperitoneal injection of $60 \mathrm{mmol} / \mathrm{L}$ chloral hydrate $(0.35 \mathrm{~g} / \mathrm{kg})$ and provided with $250 \mathrm{U} / \mathrm{kg}$ heparin through sublingual venous injection to prevent coagulation. The hearts were quickly removed and mounted on a Langendorff apparatus via the aorta for retrograde perfusion with Krebs-Henseleit $(\mathrm{K}-\mathrm{H})$ buffer at constant pressure $(10 \mathrm{KPa})$ and constant temperature $\left(37^{\circ} \mathrm{C}\right)$. The composition of $\mathrm{K}-\mathrm{H}$ buffer (in mmol/L) was as follows: $\mathrm{NaCl}, 118 ; \mathrm{KCl}, 4.7 ; \mathrm{MgSO}_{4}$, 1.2; $\mathrm{CaCl}_{2}, 2.5 ; \mathrm{KH}_{2} \mathrm{PO}_{4}, 1.2 ; \mathrm{NaHCO}_{3}, 25 ;$ glucose, 11 . The buffer was saturated with $95 \% \mathrm{O}_{2} / 5 \% \mathrm{CO}_{2}(\mathrm{pH}$ 7.4) [18]. The left atrial appendage was cut. A latex balloon filled with water was inserted into the left ventricle through the left atrial appendage. Finally, hemodynamic parameters, LVDP (LVSP is left ventricular systolic pressure; LVEDP is left ventricular end-diastolic pressure; LVDP $=$ LVSP - LVEDP), $\pm d p / d t_{\max }$ (reflecting the important indicators of left ventricular systolic function and diastolic function), CF, and HR, were displayed on the recorder screen.

2.4. Evaluation of Myocardial Infarct Size (IS). After reperfusion was concluded, the heart was frozen at $-20^{\circ} \mathrm{C}$ and cut into five slices along the transverse direction. Each piece was $2 \mathrm{~mm}$ thick. The slices were incubated in $1 \%$ triphenyltetrazolium chloride (TTC) at $37^{\circ} \mathrm{C}$ for $20 \mathrm{~min}$. The heart slices were imaged using a digital camera. Area at risk (AAR) and IS were digitally measured using Image Pro Plus software [19]. Myocardial IS was expressed as the ratio between IS and AAR.

2.5. Assay of Cellular Injury. To determine the activities of lactate dehydrogenase (LDH) and creatinine kinase (CK), the samples were collected from the coronary effluent before ischemia and after $85 \mathrm{~min}$ of reperfusion. The levels of LDH and $\mathrm{CK}$ in the effluent were detected spectrophotometrically using their corresponding cytotoxicity detection kits (Nanjing Jiancheng Biological Product, Nanjing, China).

2.6. Assay of Oxidative Stress. After perfusion, we obtained the same part of the ventricular apical. Subsequently, the tissue was homogenized in appropriate buffer and centrifuged, after which the supernatant was removed. The levels of superoxide dismutase (SOD) and malondialdehyde (MDA) and glutathione (GSH)/GSH disulfide (GSSG) ratio were analyzed spectrophotometrically using their corresponding assay kits (Nanjing Jiancheng Biological Product, Nanjing, China). 
TABLE 1: Effect of kaempferol on cardiac function in the rats subjected to I/R $(\bar{x} \pm s, \%, n=8) ;{ }^{* *} P<0.01$, compared with the control group; ${ }^{\# \#} P<0.01$, compared with the I/R group.

\begin{tabular}{|c|c|c|c|c|}
\hline Reperfusion & Control & $\mathrm{I} / \mathrm{R}$ & Kaempferol & TDZD-8 \\
\hline LVDP (mmHg) & $87.98 \pm 3.98$ & $52.43 \pm 2.62^{* *}$ & $69.47 \pm 2.26^{\# \#}$ & $64.53 \pm 2.47^{\# \#}$ \\
\hline$+\mathrm{LV} d p / d t_{\max }\left(\mathrm{mmHg} \cdot \mathrm{s}^{-1}\right)$ & $89.37 \pm 3.73$ & $45.86 \pm 2.53^{* *}$ & $63.27 \pm 2.76^{\# \#}$ & $60.58 \pm 2.96^{\# \#}$ \\
\hline$-\mathrm{LV} d p / d t_{\max }\left(\mathrm{mmHg} \cdot \mathrm{s}^{-1}\right)$ & $86.76 \pm 3.56$ & $42.16 \pm 2.38^{* *}$ & $61.54 \pm 3.14^{\# \#}$ & $60.79 \pm 3.35^{\# \#}$ \\
\hline $\mathrm{CF}\left(\mathrm{mL} \cdot \mathrm{min}^{-1}\right)$ & $82.19 \pm 3.63$ & $51.46 \pm 2.36^{* *}$ & $67.21 \pm 4.06^{\# \#}$ & $64.59 \pm 3.97^{\# \#}$ \\
\hline $\mathrm{HR}$ (beats $\cdot \min ^{-1}$ ) & $90.51 \pm 4.58$ & $70.68 \pm 4.87^{* *}$ & $83.92 \pm 3.99^{\# \#}$ & $78.68 \pm 4.13^{\# \#}$ \\
\hline
\end{tabular}

2.7. Assay of Inflammation. Tumor necrosis factor alpha (TNF- $\alpha$ ) was analyzed spectrophotometrically according to the instructions in the TNF- $\alpha$ ELISA kit (Tsz Biosciences, Greater Boston, USA).

2.8. TUNEL Assay. TUNEL assay was carried out according to the manufacturer's instructions. After deparaffinization and rehydration, the sections were treated with $10 \mathrm{mmol} / \mathrm{L}$ protease $\mathrm{K}$ for $15 \mathrm{~min}$. The slides were immersed in TUNEL reaction mixture for $60 \mathrm{~min}$ at $37^{\circ} \mathrm{C}$ in a humidified atmosphere in the dark. A converter POD was used to incubate the slides for $30 \mathrm{~min}$. The slides were then analyzed using an optical microscope. To evaluate the apoptosis index of the TUNEL-stained heart tissues, we captured 10 random fields per tissue section at 400x magnification. TUNEL index (\%) is calculated as the ratio of the number of TUNEL-positive cells divided by the total number of cells [20] (see Figure 4).

2.9. Western Blot Analysis. The protein levels of total GSK-3 $\beta$, phospho-GSK-3 $\beta$ (P-GSK-3 $\beta$, Ser9), precaspase-3, cleaved caspase- 3 , and cytoplasm cytochrome $\mathrm{C}$ were determined using Western blot analysis. After perfusion by Langendorff apparatus, we cut off the same part in ventricular apical of the rats, then homogenized the cut tissue in appropriate buffer, and centrifuged it. Supernatant was extracted and boiled for $15 \mathrm{~min}$ to make protein denaturation. Then the whole-cell protein extracts were separated using 12\% SDSpolyacrylamide gel electrophoresis. Proteins were transferred to nylon membranes by electrophoretic transfer system. The membranes were blocked with 5\% skimmed milk blocking buffer at room temperature for $1 \mathrm{~h}$ and then incubated with primary antibodies overnight $(18 \mathrm{~h})$ at $4^{\circ} \mathrm{C}$. After being washed with TBST buffer, the corresponding secondary antibodies were used to identify primary antibody binding. In the end, the blots were visualized with ECL-plus reagent.

2.10. Statistical Analysis. Results were expressed as mean \pm S.D. and analyzed using one-way analysis of variance. The values with $P<0.05$ were considered statistically significant. All statistical tests were performed with GraphPad Prism software version 5.0 (GraphPad Software, San Diego, CA).

\section{Results}

3.1. Kaempferol Improved the Recovery of I/R-Induced Cardiac Function. As shown in Table 1, the hearts in the I/R group demonstrated significant decrease in the recovery of cardiac
TABLE 2: Effect of kaempferol on the levels of CK and LDH in the coronary effluent before ischemia and after $85 \mathrm{~min}$ of reperfusion $(\bar{x} \pm s, n=8) ;{ }^{* *} P<0.01$, compared with the control group; ${ }^{\# *} P<$ 0.01 , compared with the I/R group.

\begin{tabular}{lcc}
\hline Groups & Before ischemia & Reperfusion \\
\hline CK (U/L) & & \\
Control & $16.73 \pm 1.46$ & $24.81 \pm 1.31$ \\
I/R & $15.76 \pm 1.21$ & $56.74 \pm 2.97^{* *}$ \\
Kaempferol & $16.72 \pm 1.23$ & $32.30 \pm 2.48^{\# \#}$ \\
TDZD-8 & $16.45 \pm 1.26$ & $36.73 \pm 2.54^{\# \#}$ \\
LDH (U/L) & & \\
Control & $14.47 \pm 1.36$ & $19.51 \pm 1.35$ \\
I/R & $14.58 \pm 1.38$ & $60.54 \pm 2.35^{* *}$ \\
Kaempferol & $15.35 \pm 0.96$ & $36.63 \pm 1.83^{\# \#}$ \\
TDZD-8 & $15.49 \pm 1.12$ & $39.67 \pm 1.64^{\# \#}$ \\
\hline
\end{tabular}

function compared with that in the control group. Moreover, the hearts in the kaempferol and TDZD- 8 treatment groups showed higher recovery of cardiac function than that in the I/R group $(P<0.05)$. Hemodynamic data confirmed that kaempferol improved the recovery of the cardiac systolic and diastolic function of the rats after $\mathrm{I} / \mathrm{R}$.

3.2. Kaempferol Reduced Myocardial IS Post-I/R. The representative slices of the hearts are shown in Figure 1(a). The ratio of IS and AAR in the control group was $5.31 \% \pm$ $1.34 \%$, which was significantly different from that of the I/R group $(46.73 \% \pm 1.88 \%)(P<0.01$; Figure $1(\mathrm{~b}))$. The ratio remarkably decreased in the kaempferol $(16.49 \% \pm 1.23 \%)$ and TDZD-8 groups $(21.42 \% \pm 1.48 \%)$ compared with that in the I/R group $(P<0.01$; Figure $1(\mathrm{~b}))$.

3.3. Kaempferol Attenuated the I/R-Induced Enzyme Release in Rat Heart. As shown in Table 2, the levels of perfusate $\mathrm{CK}$ and $\mathrm{LDH}$ in the I/R group $(56.74 \pm 2.97$ and $60.54 \pm$ 2.35 , resp.) significantly increased compared with that in the control group $(P<0.01)$; however, the levels of CK and $\mathrm{LDH}$ in the kaempferol $(32.30 \pm 2.48$ and $36.63 \pm 1.83$, resp.) and TDZD- 8 groups $(36.73 \pm 2.54$ and $39.67 \pm 1.64$, resp.) $(P<0.01)$ were significantly decreased compared with the I/R group.

3.4. Kaempferol Improved the Oxidative Stress State Induced by $I / R$. To identify the possible mechanisms of kaempferol involving antioxidants on cardioprotection, we evaluated 


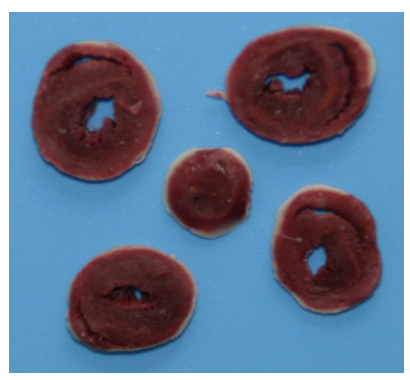

Control

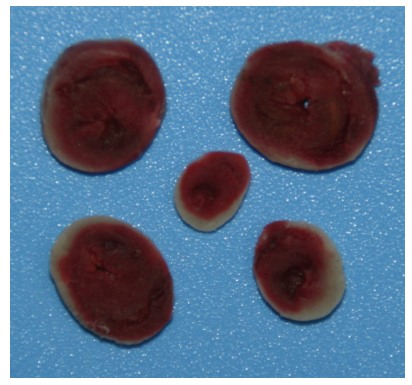

Kaempferol

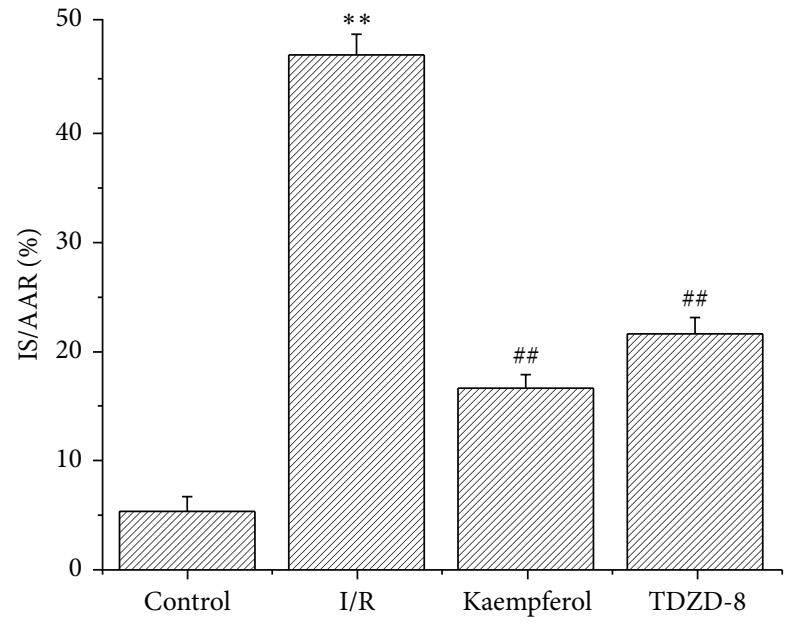

(b)

FIGURE 1: Kaempferol reduces the I/R-induced IS. (a) Images of myocardial tissue sections after TTC staining. (b) The ratio between IS and AAR, in which AAR is the area at risk and IS is the infarct size; values are presented as means with their standard deviations $(\bar{x} \pm s$, $n=8) ;{ }^{* *} P<0.01$, compared with the control group; ${ }^{\# \#} P<0.01$, compared with the I/R group.

SOD activity, MDA level, and GSH/GSSG ratio in the myocardial tissues. As shown in Table 3, and Figure 2, the SOD activity and the ratio of GSH/GSSG in the kaempferol or TDZD-8 groups significantly increased, compared with those of the control group, whereas the MDA level significantly decreased in the kaempferol or TDZD- 8 groups.

3.5. Kaempferol Reduced the Inflammatory Response. As shown in Figure 3, the level of TNF- $\alpha$ increased from $95 \pm$ $4.5 \mathrm{pg} / \mathrm{mL}$ in the control group to $328 \pm 16.7 \mathrm{pg} / \mathrm{mL}$ in

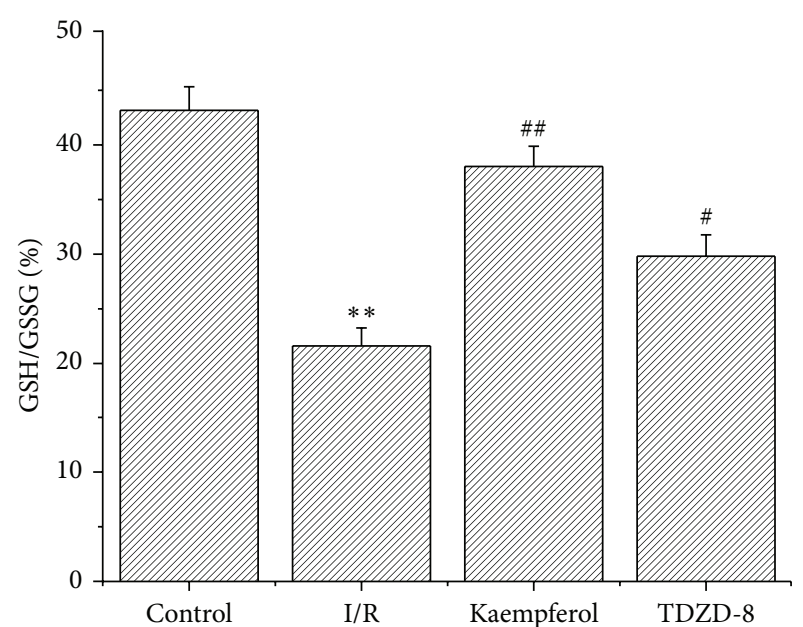

FIGURE 2: Kaempferol increases the ratio of GSH/GSSG. Values are presented as means with their standard deviation $(\bar{x} \pm s, n=8)$; ${ }^{* *} P<0.01$, compared with control group; ${ }^{\# \#} P<0.01$ and ${ }^{\#} P<0.05$, compared with the I/R group.

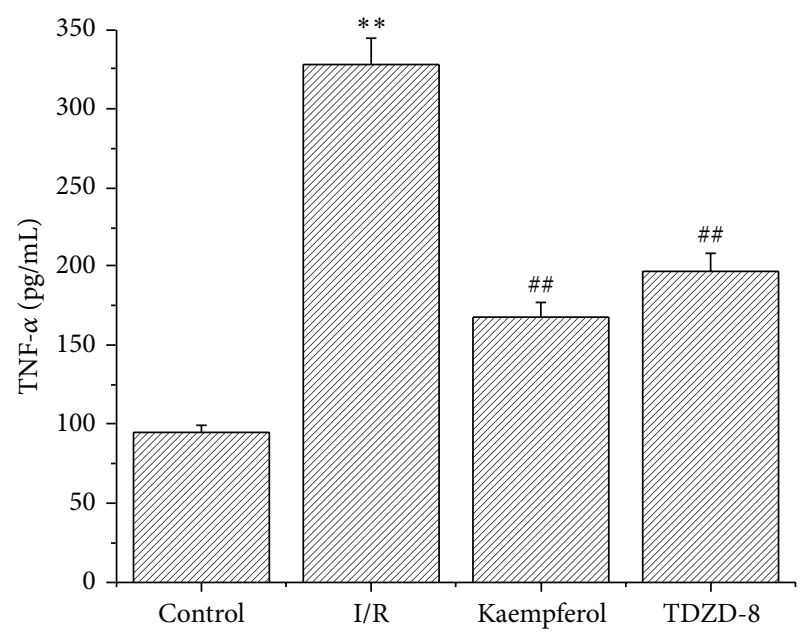

FIGURE 3: Kaempferol reduces inflammatory response. Values are presented as means with their standard deviations $(\bar{x} \pm s, n=8)$; ${ }^{* *} P<0.01$, compared with the control group; ${ }^{\# \#} P<0.01$, compared with the I/R group.

the I/R group. The levels of TNF- $\alpha$ in the kaempferol $(168 \pm 9.3 \mathrm{pg} / \mathrm{mL})$ and TDZD-8 groups $(197 \pm 11.4 \mathrm{pg} / \mathrm{mL})$ significantly decreased compared with that in the I/R group $(P<0.01)$.

3.6. Kaempferol Weakened the Cardiomyocyte Apoptosis Induced by $I / R$. TUNEL staining did not show a significant apoptotic phenomenon in the control group, whereas the number of apoptotic cells evidently increased in the I/R group $(58 \pm 3.21)$. Apoptotic cells significantly decreased in the kaempferol $(35 \pm 1.48)$ and TDZD-8 groups $(47 \pm 2.79)$ compared with those in the I/R group $(P<0.01)$. 
TABLE 3: Effects of kaempferol on SOD activity and MDA level. Values are presented as means with their standard deviations $(\bar{x} \pm s$, $n=8) ;{ }^{* *} P<0.01$, compared with the control group; ${ }^{\# \#} P<0.01$ and ${ }^{\#} P<0.05$, compared with the I/R group.

\begin{tabular}{lccc}
\hline \multirow{2}{*}{ Group } & \multirow{2}{*}{ Dosage } & \multicolumn{2}{c}{ Reperfusion } \\
& & SOD (U/mgPr) & MDA (nmol/mgPr) \\
\hline Control & - & $10.48 \pm 0.79$ & $210.43 \pm 12.56$ \\
I/R & - & $3.35 \pm 0.41^{* *}$ & $489.71 \pm 30.25^{* *}$ \\
Kaempferol & $15 \mathrm{mmol} / \mathrm{L}$ & $8.15 \pm 0.57^{\# \#}$ & $285.76 \pm 19.04^{\# \#}$ \\
TDZD-8 & $0.01 \mathrm{mmol} / \mathrm{L}$ & $5.43 \pm 0.62^{\#}$ & $370.24 \pm 22.50^{\# \#}$ \\
\hline
\end{tabular}

3.7. Effects of Kaempferol on GSK-3 $\beta$ Phosphorylation, Cytochrome C Release, and Caspase-3 Activity. As shown in Figure 5, the level of GSK-3 $\beta$ phosphorylation in the kaempferol $(0.65 \pm 0.039)$ and TDZD-8 groups $(0.74 \pm 0.043)$ significantly increased compared with those in the control $(0.32 \pm 0.018)$ and $\mathrm{I} / \mathrm{R}$ groups $(0.35 \pm 0.02)(P<0.01)$. However, no evident difference was observed between the control and I/R groups. The release of cytochrome $\mathrm{C}$ and the dissociation of caspase- 3 in the I/R groups $(0.52 \pm 0.039$ and $0.37 \pm 0.021$, resp.) were significantly increased compared with those in the control groups $(P<0.01)$, whereas the kaempferol $(0.32 \pm 0.024$ and $0.25 \pm 0.018$, resp.) and TDZD-8 groups $(0.21 \pm 0.013$ and $0.29 \pm 0.019$, resp.) were significantly decreased compared with those in the I/R groups $(P<0.01)$.

\section{Discussion}

In this study, we investigated the effects of kaempferol on cardiac function, myocardial IS, cardiomyocyte apoptosis, inflammation factor, and myocardial enzyme in the isolated rat heart model of I/R. We provided evidence that kaempferol improves the recovery of cardiac function, reduces intracellular oxidation status and myocardial IS, and inhibits myocardial apoptosis induced by I/R. Finally, we demonstrated that the phosphorylation of GSK- $3 \beta$ is involved in the cardioprotection of kaempferol.

Reactive oxygen species (ROS) induced injury plays an important role in the development of $\mathrm{I} / \mathrm{R}$ in various organs [21]. Few free radicals are present under physiological conditions during ischemia; thus, the absorbance of oxygen radical decreases [22]. During recovery, the blood supply of the tissues triggers the "explosion" of oxygen free radicals; hence, the accumulated ROS attack the cells and cause damage [23]. ROS causes damage to the cell membranes in rat heart, subsequently causes cell membrane lipid peroxidation and structural failure, and results in leakage of myocardial enzyme. Inhibiting the ROS generation or the antagonist of reactive oxygen toxicity is important to alleviate myocardial reperfusion injury [24]. In the present experiment, SOD activity and GSH/GSSG ratio significantly increased, whereas MDA level significantly decreased in the kaempferol group. Therefore, we speculated that kaempferol has good antioxidant effect that functions as myocardial protection.

During $I / R$, oxidative stress activates NF- $\kappa B$ because cardiac stress rapidly responds to the expressed genes. NF- $\kappa \mathrm{B}$

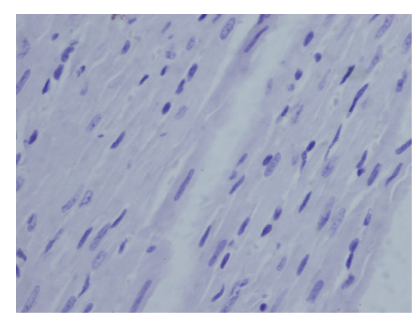

Control

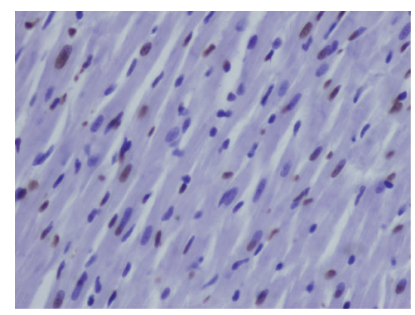

Kaempferol

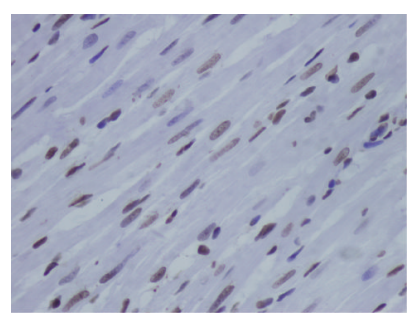

$\mathrm{I} / \mathrm{R}$

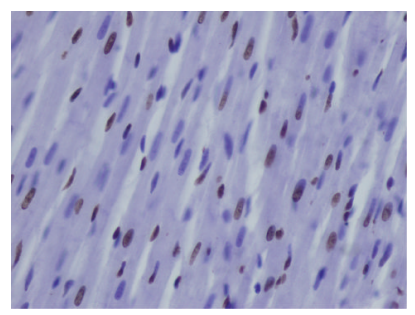

TDZD-8 (a)

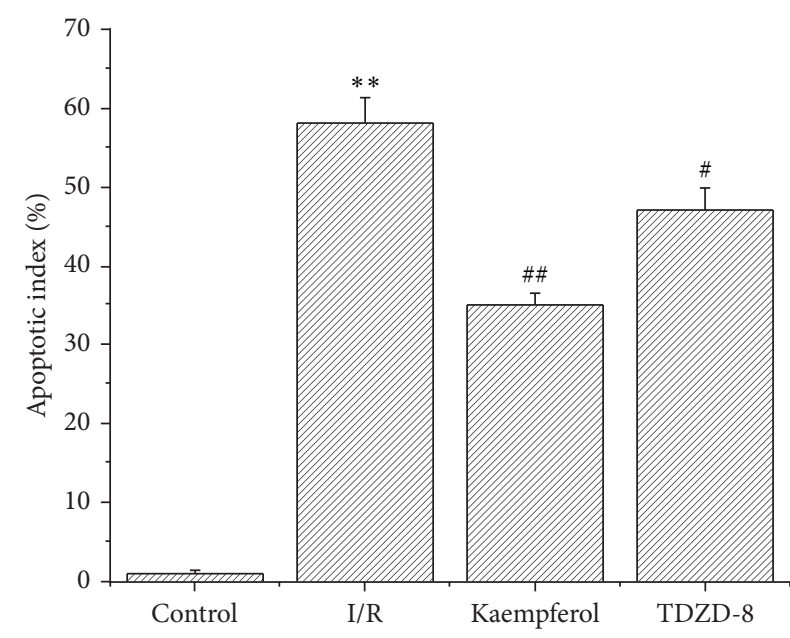

(b)

FIGURE 4: Kaempferol prevents myocardial cell apoptosis in I/R. (a) Cell apoptosis was analyzed using TUNEL staining. Magnification: 400x. (b) Apoptosis cells/total cells (\%). Values are presented as means with their standard deviations $(\bar{x} \pm s, n=8) ;{ }^{* *} P<0.01$, compared with the control group; ${ }^{\# \#} P<0.01$ and ${ }^{\#} P<0.05$, compared with the I/R group.

stimulates the cardiac cells and macrophages and produces large amounts of TNF- $\alpha$; thus, the myocardial cells after I/R are the sites for TNF- $\alpha$ synthesis and the target organs of TNF- $\alpha$. Studies have shown that TNF- $\alpha$ dose dependently decreases myocardial contractility, whereas the application of anti-TNF- $\alpha$ reduces I/R injury and confers protection for the ischemic myocardium [25]. The TNF- $\alpha$ bioactivity in the heart increases from the early stage of I/R; such increase has been speculated to partially contribute to the increased area of myocardial infarction [26]. The ROS generated within cells can induce apoptosis through multiple pathways. Cardiomyocytes, which are abundant in the mitochondria, are the major endogenous source and are the susceptible target 


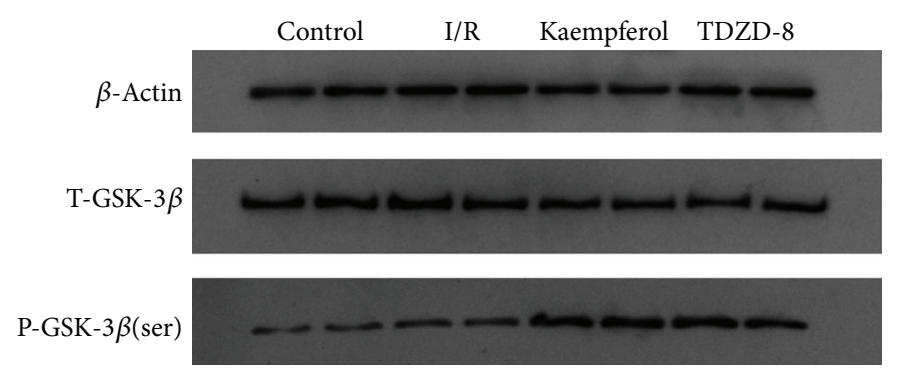

(a)

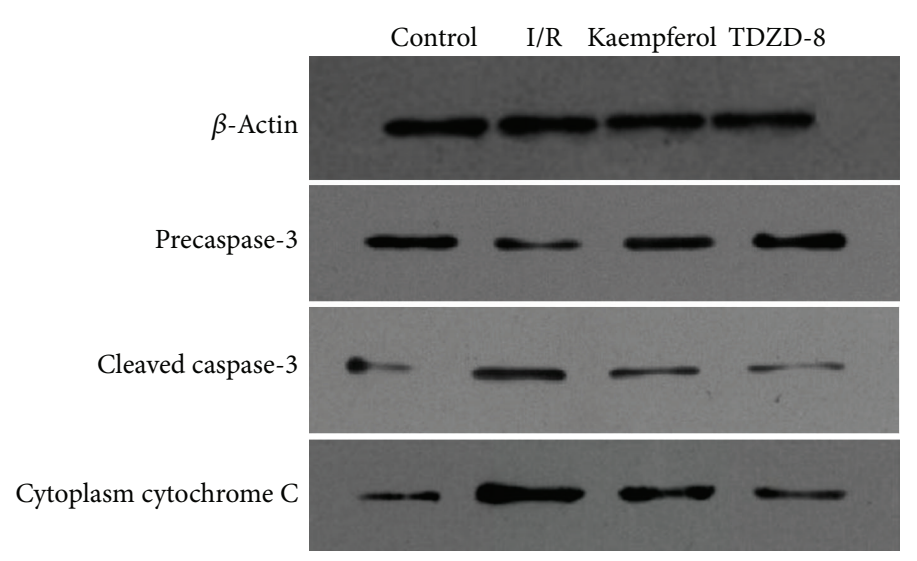

(c)

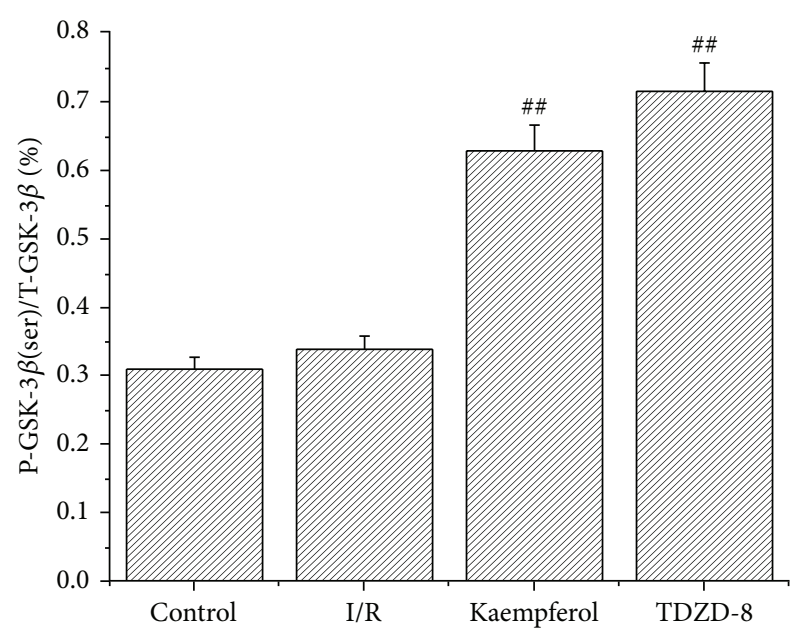

(b)

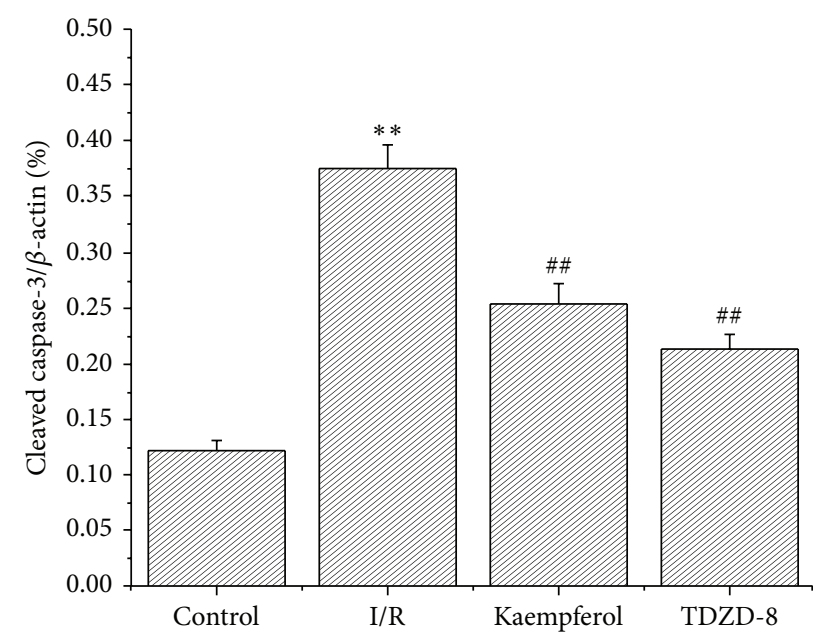

(d)

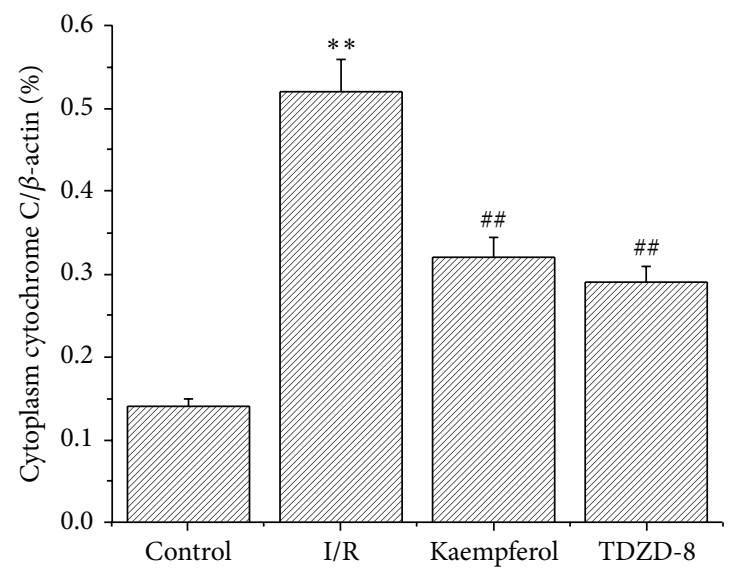

(e)

FIGURE 5: Kaempferol increases the phosphorylation of GSK-3 $\beta$ and reduces the release of cytochrome $\mathrm{C}$ and the dissociation of caspase-3. (a) Representative Western blots for T-GSK-3 $\beta$ and P-GSK-3 $\beta$ (Ser9). Lanes 1 and 2 are the control group, lanes 3 and 4 are the I/R group, lanes 5 and 6 are the kaempferol group, and lanes 7 and 8 are the TDZD- 8 group. (b) Grey value analysis demonstrates that kaempferol increases the ratio of P-GSK-3 $\beta$ (ser)/T-GSK-3 $\beta$. (c) Representative Western blot for cleaved caspase- 3 and cytoplasm cytochrome C. Lane 1 is the control group, lane 2 is the I/R group, lane 3 is the kaempferol group, and lane 4 is the TDZD- 8 group. (d) Grey value analysis demonstrates that kaempferol reduces the ratio of cleaved caspase-3/ $\beta$-actin. (e) Grey value analysis demonstrates that kaempferol reduces the ratio of cytoplasm cytochrome $\mathrm{C} / \beta$-actin. Values are presented as means with their standard deviations $(\bar{x} \pm s, n=8) ;{ }^{* *} P<0.01$, compared with the control group; ${ }^{\# \#} P<0.01$, compared with the I/R group. 
of ROS damage [27]; the abundant ROS within the mitochondria can decrease its selective ion permeability or change its membrane permeability, which changes the mitochondrial membrane and thereby induces mitochondrial permeability transition pore (MPTP) opening during reperfusion [28]. The mitochondria release cytochrome $\mathrm{C}$, which activates the aspartate-specific cysteine proteases (caspases) and induces apoptosis [29].

Cytochrome $\mathrm{C}$ is located within the intermembrane space under normal conditions; the released cytochrome $\mathrm{C}$ binds to the C-terminal domain of the apoptotic protease activating factor-1 (Apaf-1) and changes its conformation [30]. The activated Apaf-1/cytochrome C complex promotes caspase activation [31]. Several studies in the literature showed that GSK-3 $\beta$ inhibition might delay or suppress mPTP opening and inhibit the release of cytochrome $C[32,33]$. TDZD-8 is a GSK- $3 \beta$ inhibitor with significant myocardial protection effect via inhibition of inflammation and apoptosis [34]. We used TDZD- 8 as a positive control agent to demonstrate the mechanisms of the cardioprotective effects of kaempferol. Our research showed that kaempferol or TDZD-8 can increase the level of GSK- $3 \beta$ phosphorylation and reduce the release of cytochrome $\mathrm{C}$ compared with those in the control and I/R groups. We inferred that kaempferol can function similarly to that of TDZD- 8 on GSK- $3 \beta$ phosphorylation. Thus, we speculated that kaempferol decreases the apoptosis induced by I/R injury via GSK-3 $\beta$ inhibition.

\section{Conflict of Interests}

No conflict of interests, financial or otherwise, is declared by the authors.

\section{Authors' Contribution}

Dong Wang and Mingjie Zhou conceived and designed the experiments. Mingjie Zhou and Qiusheng Zheng performed the experiments. Wenjuan Wang, Jichun Han, and Huanhuan Ren analyzed the data. Wenjuan Wang, Jichun Han, and Huanhuan Ren contributed reagents/materials/analysis tools. Mingjie Zhou wrote the paper.

\section{Acknowledgments}

This study was supported by the Natural Science Foundation of Shandong Province (no. ZR2011HL013) to Dong Wang and by the Science and Technology Foundation of Shihezi (2014QY16) to Qiusheng Zheng.

\section{References}

[1] Q. Fan, L. Chen, S. Cheng et al., "Aging aggravates nitratemediated ROS/RNS changes," Oxidative Medicine and Cellular Longevity, vol. 2014, Article ID 376515, 7 pages, 2014.

[2] M. Juhaszova, D. B. Zorov, Y. Yaniv, H. B. Nuss, S. Wang, and S. J. Sollott, "Role of glycogen synthase kinase- $3 \beta$ in cardioprotection," Circulation Research, vol. 104, no. 11, pp. 1240-1252, 2009.
[3] R. B. Jennings, "Historical perspective on the pathology of myocardial ischemia/reperfusion injury," Circulation Research, vol. 113, no. 4, pp. 428-438, 2013.

[4] K. Przyklenk, Y. Dong, V. V. Undyala, and P. Whittaker, "Autophagy as a therapeutic target for ischaemia/reperfusion injury? Concepts, controversies, and challenges," Cardiovascular Research, vol. 94, no. 2, pp. 197-205, 2012.

[5] H. Liu, J. Wang, W. Zhou, Y. Wang, and L. Yang, "Systems approaches and polypharmacology for drug discovery from herbal medicines: an example using licorice," Journal of Ethnopharmacology, vol. 146, no. 3, pp. 773-793, 2013.

[6] A. Ali, K. P. Hoeflich, and J. R. Woodgett, "Glycogen synthase kinase-3: properties, functions, and regulation," Chemical Reviews, vol. 101, no. 8, pp. 2527-2540, 2001.

[7] S. H. Ramirez, S. Fan, M. Zhang et al., "Inhibition of glycogen synthase kinase $3 \beta$ (GSK3 $\beta$ ) decreases inflammatory responses in brain endothelial cells," The American Journal of Pathology, vol. 176, no. 2, pp. 881-892, 2010.

[8] S. Cuzzocrea, C. Crisafulli, E. Mazzon et al., "Inhibition of glycogen synthase kinase- $3 \beta$ attenuates the development of carrageenan-induced lung injury in mice," British Journal of Pharmacology, vol. 149, no. 6, pp. 687-702, 2006.

[9] P. H. Sugden, S. J. Fuller, S. C. Weiss, and A. Clerk, "Glycogen synthase kinase 3 (GSK3) in the heart: a point of integration in hypertrophic signalling and a therapeutic target? A critical analysis," British Journal of Pharmacology, vol. 153, no. S1, pp. S137-S153, 2008.

[10] A. Mora, K. Sakamoto, E. J. McManus, and D. R. Alessi, "Role of the PDK1-PKB-GSK3 pathway in regulating glycogen synthase and glucose uptake in the heart," FEBS Letters, vol. 579, no. 17, pp. 3632-3638, 2005.

[11] M. Juhaszova, D. B. Zorov, S.-H. Kim et al., "Glycogen synthase kinase- $3 \beta$ mediates convergence of protection signalling to inhibit the mitochondrial permeability transition pore," Journal of Clinical Investigation, vol. 113, no. 11, pp. 1535-1549, 2004.

[12] M. Nishihara, T. Miura, T. Miki et al., "Modulation of the mitochondrial permeability transition pore complex in GSK$3 \beta$-mediated myocardial protection," Journal of Molecular and Cellular Cardiology, vol. 43, no. 5, pp. 564-570, 2007.

[13] W. Ren, Z. Qiao, H. Wang, L. Zhu, and L. Zhang, "Flavonoids: promising anticancer agents," Medicinal Research Reviews, vol. 23, no. 4, pp. 519-534, 2003.

[14] M. López-Lázaro, "Distribution and biological activities of the flavonoid luteolin," Mini-Reviews in Medicinal Chemistry, vol. 9, no. 1, pp. 31-59, 2009.

[15] A. R. Verma, M. Vijayakumar, C. S. Mathela, and C. V. Rao, "In vitro and in vivo antioxidant properties of different fractions of Moringa oleifera leaves," Food and Chemical Toxicology, vol. 47, no. 9, pp. 2196-2201, 2009.

[16] M. J. Park, E. K. Lee, H.-S. Heo et al., “The anti-inflammatory effect of kaempferol in aged kidney tissues: the involvement of nuclear factor- $\kappa \mathrm{B}$ via nuclear factor-inducing kinase $/ \mathrm{I} \kappa \mathrm{B}$ kinase and mitogen-activated protein kinase pathways," Journal of Medicinal Food, vol. 12, no. 2, pp. 351-358, 2009.

[17] M. A. Gates, S. S. Tworoger, J. L. Hecht, I. de Vivo, B. Rosner, and S. E. Hankinson, "A prospective study of dietary flavonoid intake and incidence of epithelial ovarian cancer," International Journal of Cancer, vol. 121, no. 10, pp. 2225-2232, 2007. 
[18] J. L. Zweier, J. Fertmann, and G. Wei, "Nitric oxide and peroxynitrite in postischemic myocardium," Antioxidants \& Redox Signaling, vol. 3, no. 1, pp. 11-22, 2001.

[19] Y. Wang, P. Xu, H. Liu, Y. Zhou, and X. Cao, “The protection of salidroside of the heart against acute exhaustive injury and molecular mechanism in rat," Oxidative Medicine and Cellular Longevity, vol. 2013, Article ID 507832, 8 pages, 2013.

[20] H. Pang, B. Han, T. Yu, and Z. Peng, "The complex regulation of tanshinone IIA in rats with hypertension-induced left ventricular hypertrophy," PLoS ONE, vol. 9, no. 3, Article ID e92216, 2014.

[21] Z. Xia, Y. Chen, Q. Fan, and M. Xue, "Oxidative stressmediated reperfusion injury: mechanism and therapies," Oxidative Medicine and Cellular Longevity, vol. 2014, Article ID 373081, 2 pages, 2014.

[22] M. Valko, D. Leibfritz, J. Moncol, M. T. D. Cronin, M. Mazur, and J. Telser, "Free radicals and antioxidants in normal physiological functions and human disease," International Journal of Biochemistry and Cell Biology, vol. 39, no. 1, pp. 44-84, 2007.

[23] W. Dröge, "Free radicals in the physiological control of cell function," Physiological Reviews, vol. 82, no. 1, pp. 47-95, 2002.

[24] Y. Wang, X. Li, X. Wang et al., "Ginsenoside Rd attenuates myocardial ischemia/reperfusion injury via Akt/GSK-3 $\beta$ signaling and inhibition of the mitochondria-dependent apoptotic pathway," PLoS ONE, vol. 8, no. 8, Article ID e70956, 2013.

[25] B. S. Cain, D. R. Meldrum, C. A. Dinarello et al., "Tumor necrosis factor- $\alpha$ and interleukin- $1 \beta$ synergistically depress human myocardial function," Critical Care Medicine, vol. 27, no. 7, pp. 1309-1318, 1999.

[26] M. Sugano, T. Hata, K. Tsuchida et al., "Local delivery of soluble TNF-alpha receptor 1 gene reduces infarct size following ischemia/reperfusion injury in rats," Molecular and Cellular Biochemistry, vol. 266, no. 1-2, pp. 127-132, 2004.

[27] P. Korge, P. Ping, and J. N. Weiss, "Reactive oxygen species production in energized cardiac mitochondria during hypoxia/reoxygenation: modulation by nitric oxide," Circulation Research, vol. 103, no. 8, pp. 873-880, 2008.

[28] T. Miura and T. Miki, "GSK-3 $\beta$, a therapeutic target for cardiomyocyte protection," Circulation Journal, vol. 73, no. 7, pp. 1184-1192, 2009.

[29] A. Wyllie, "Clues in the p53 murder mystery," Nature, vol. 389, no. 6648 , pp. 237-238, 1997.

[30] P. Korge, H. M. Honda, and J. N. Weiss, "Protection of cardiac mitochondria by diazoxide and protein kinase C: implications for ischemic preconditioning," Proceedings of the National Academy of Sciences of the United States of America, vol. 99, no. 5, pp. 3312-3317, 2002.

[31] V. Borutaite, A. Jekabsone, R. Morkuniene, and G. C. Brown, "Inhibition of mitochondrial permeability transition prevents mitochondrial dysfunction, cytochrome c release and apoptosis induced by heart ischemia," Journal of Molecular and Cellular Cardiology, vol. 35, no. 4, pp. 357-366, 2003.

[32] S.-S. Park, H. Zhao, Y. Jang, R. A. Mueller, and Z. Xu, “ $N^{6}-(3-$ Iodobenzyl)-adenosine- $5^{\prime}-N$-methylcarboxamide confers cardioprotection at reperfusion by inhibiting mitochondrial permeability transition pore opening via glycogen synthase kinase $3 \beta$," Journal of Pharmacology and Experimental Therapeutics, vol. 318, no. 1, pp. 124-131, 2006.
[33] J. Feng, E. Lucchinetti, P. Ahuja, T. Pasch, J.-C. Perriard, and M. Zaugg, "Isoflurane postconditioning prevents opening of the mitochondrial permeability transition pore through inhibition of glycogen synthase kinase $3 \beta$," Anesthesiology, vol. 103, no. 5, pp. 987-995, 2005.

[34] H. K. Gao, Z. Yin, N. Zhou, X. Y. Feng, F. Gao, and H. C. Wang, "Glycogen synthase kinase 3 inhibition protects the heart from acute ischemia-reperfusion injury via inhibition of inflammation and apoptosis," Journal of Cardiovascular Pharmacology, vol. 52, no. 3, pp. 286-292, 2008. 


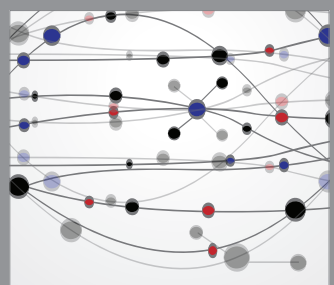

The Scientific World Journal
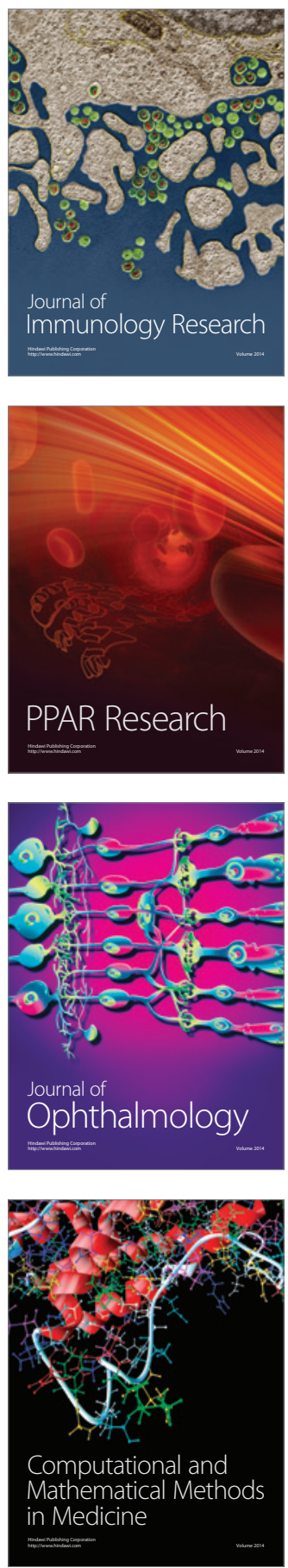

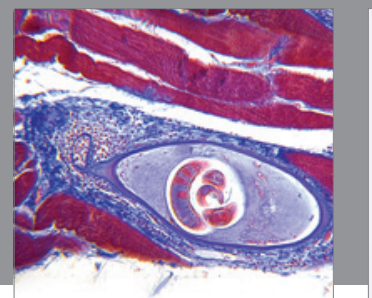

Gastroenterology

Research and Practice
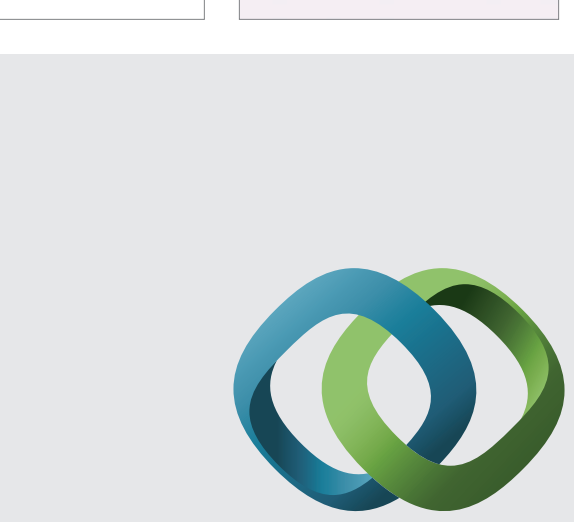

\section{Hindawi}

Submit your manuscripts at

http://www.hindawi.com
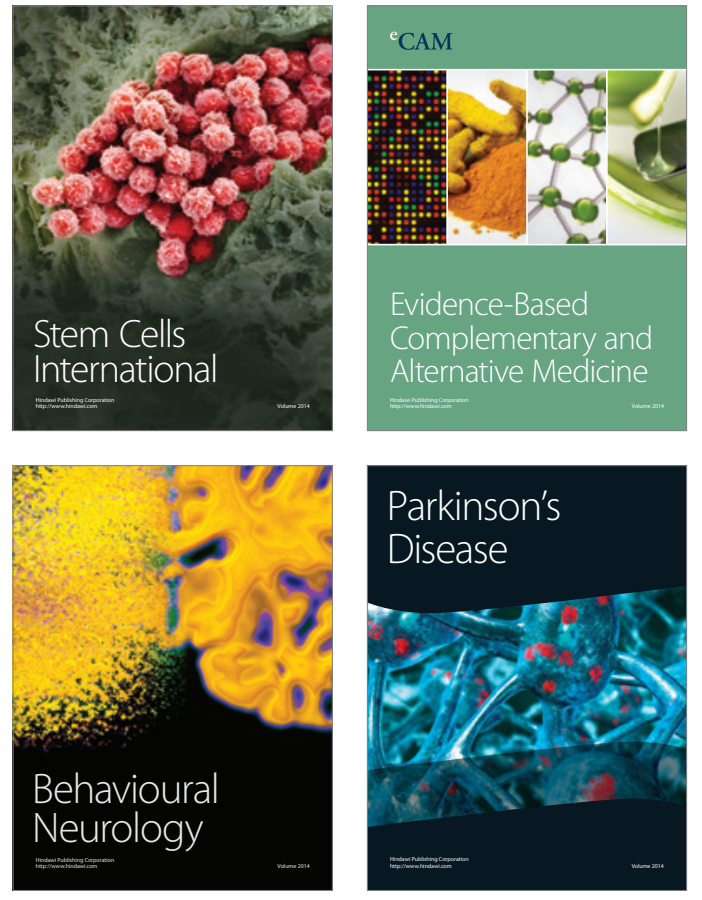
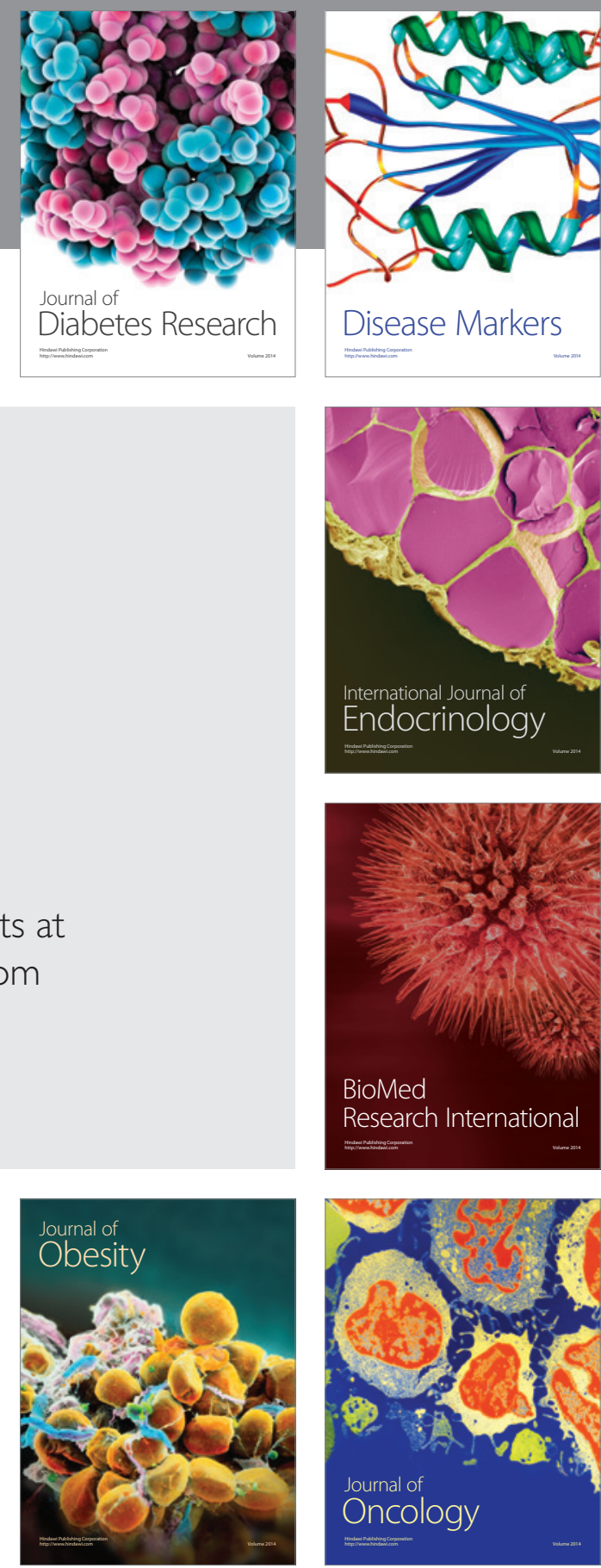

Disease Markers
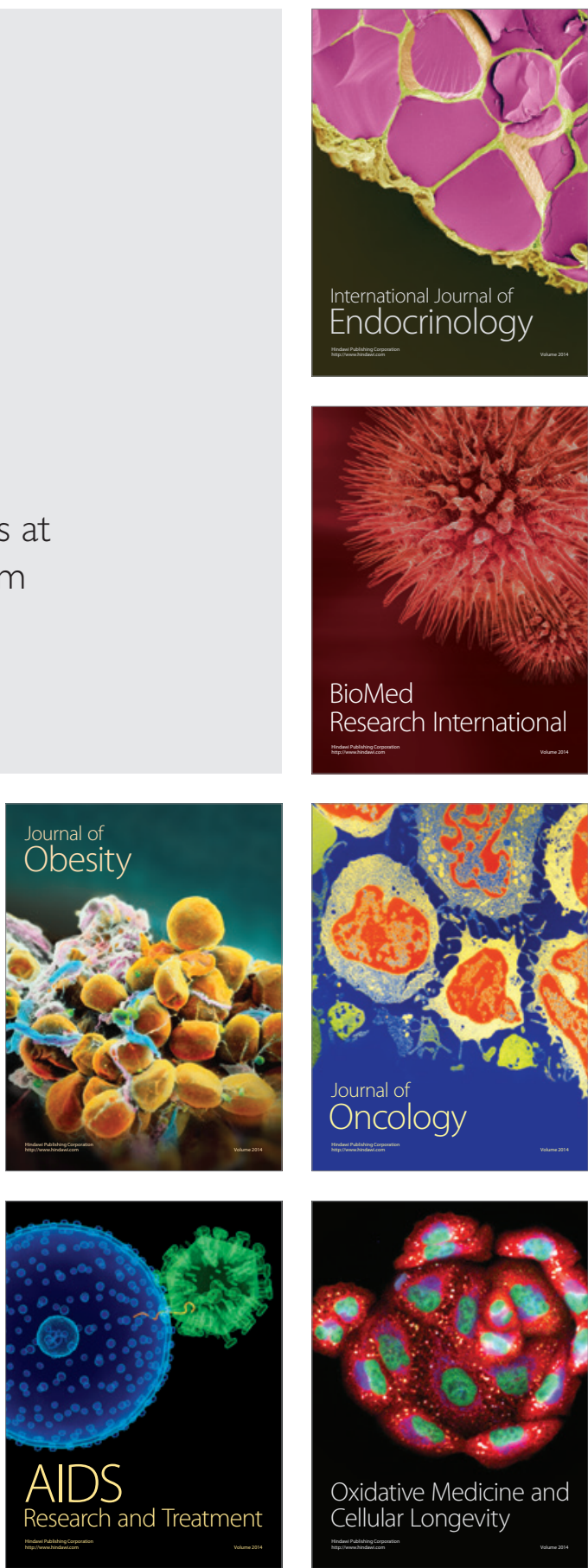